\title{
Soliton and ratchet motions in helices
}

\author{
A.V.Zolotaryuk ${ }^{1,2}$, P.L.Christiansen ${ }^{2}$, B.Nordén ${ }^{3}$, \\ A.V.Savin ${ }^{2,4}$ \\ 1 Bogolyubov Institute for Theoretical Physics, 252143 Kyiv, Ukraine \\ 2 Department of Mathematical Modelling, Technical University of Denmark, \\ DK-2800 Lyngby, Denmark \\ 3 Department of Physical Chemistry, Chalmers University of Technology, \\ S-412 96 Gothenburg, Sweden \\ 4 State Institute for Problems of Physics and Technology, 119034 Moscow, \\ The Russian Federation
}

Received September 4, 1998

The most simple and straightforward generalization of the standard onedimensional molecular chain with nearest-neighbour interactions to three dimensions is shown to be a helix backbone with three types of intermolecular interactions, the chain with secondary structure (e.g., $\alpha$-helix in biology). The set of coupled nonlinear field equations with respect to the longitudinal and transverse displacements of the chain molecules is derived and stable non-topological three-component soliton solutions are found numerically. These solutions describe supersonic pulses of longitudinal compression accompanied by a localized transverse bulge and a localized torsional stretching of the helix backbone. Asymmetry caused by helicity of the backbone is shown to rectify zero-mean oscillating or fluctuating motions on a simple experimental mechanical model.

Key words: anharmonic chains, helices, solitons, ratchets

PACS: $63.20 . R y, 63.20 . P w, 87.15 .-v$

\section{Introduction}

The standard one-dimensional (1D) nonlinear (i.e., anharmonic) lattice, the studies of which were originated in a series of pioneering works [1-3], is usually considered as the basic model for describing the transport properties in biomolecules [4-6]. The interatomic (or intermolecular) interaction potential of this 1D lattice (linear chain) contains a hard anharmonicity and, as a result, dynamically stable solitary waves (called lattice or acoustic solitons) can propagate along the chain with supersonic velocities $[2,3]$. Recently, this chain is also referred to as the Fermi-Pasta-Ulam (FPU) chain, being currently an object for extensive studies of nonlinear dynamics. However, in applications to real biological macromolecules [7], the 1D FPU model should 
be generalized in order to include transverse motions of chain molecules [8], resulting in the appearance of secondary structure of the chain - the fundamental property of biomolecular structure.

In the present paper we discuss the most simple way in which such a generalization, that leads to secondary structure, can be accomplished. We assume that all the chain molecules are allowed to move in the 3D space. Next, all intermolecular interactions are supposed to be of the point-point type. Such a chain will be stabilized only if its structure takes the form of a helix backbone. This backbone is considered to be an isolated object which is not subject to any substrate potential. The interactions between the first- and second-neighbour molecules along the linear chain, as well as the nearest-neighbour molecules that appear in the direction of the helix backbone, provide the stability of such a structure in three dimensions. Particularly, for the $\alpha$-helix macromolecule, these interactions form the bonds between the first, second, and third neighbours.

The essential ingredient for the existence of the acoustic solitons is the hard anharmonicity of the forces that provide secondary structure. However, due to the geometry of helical structure, an effective (geometrical) anharmonicity appears that counterbalances the effect of forming the acoustic solitons. We show that only if the intrinsic (hard) anharmonicity exceeds a certain critical magnitude, the solitons are formed. For breather excitations the geometric nonlinearity is known to favour these localized modes as shown previously [9].

Inspired by Feynman's thermal ratchet [10], there have been considerable efforts recently $[11,12]$ in creating and studying devices that can act as molecular motors. The main condition for these devices functioning as thermal ratchets, is broken spatial symmetry of the system. Intuitively, one can expect that the helical asymmetry can rectify a zero-mean deterministic or stochastic rotatory motion due to right- or left-handed structure in a similar way. To this end, we have constructed a simple experimental setup that clearly demonstrates unidirectional rotary movement.

\section{Helical structure}

Let molecules (e.g., amino acids) be linked together in a molecular (polypeptide) chain by the first-, second-, and $\nu$-neighbour forces as shown in figure 1 . The forces between the $n$th and $(n+\nu)$ th molecules are assumed to form a soft (hydrogen) bond of the helix backbone and therefore the integer $\nu$ (i.e., the number of backbone spines) is determined from the condition that the $\nu$-neighbour bond has the shortest length [13]. The geometry of a regular helix backbone, when its molecules are found in the equilibrium positions, can be uniquely given by a set of three parameters [13]. We define the positions of the vertices of the helix backbone in terms of (i) the radius $R_{0}$ of the cylinder that spans the backbone, (ii) the angle $\phi$ in the $X Y$ plane which is formed by each three successive chain molecules (this angle is the projection of the valence angle $\psi$ between the nearest valence bonds onto the $X Y$ plane), and (iii) the height $\Delta z$ which measures the $Z$ projection of the distance between the nearest-neighbour molecules in the chain. Then the $X, Y$, and $Z$ coordinates of the 
radius-vector of the $n$th molecule can be written as follows: $R_{0} \cos (n \phi), R_{0} \sin (n \phi)$, and $n \Delta z$ where $n=0, \pm 1, \ldots$ When $\phi>2 \pi / \nu$ or $\phi<2 \pi / \nu$, we refer to such a chain as a right- or left-handed helix.

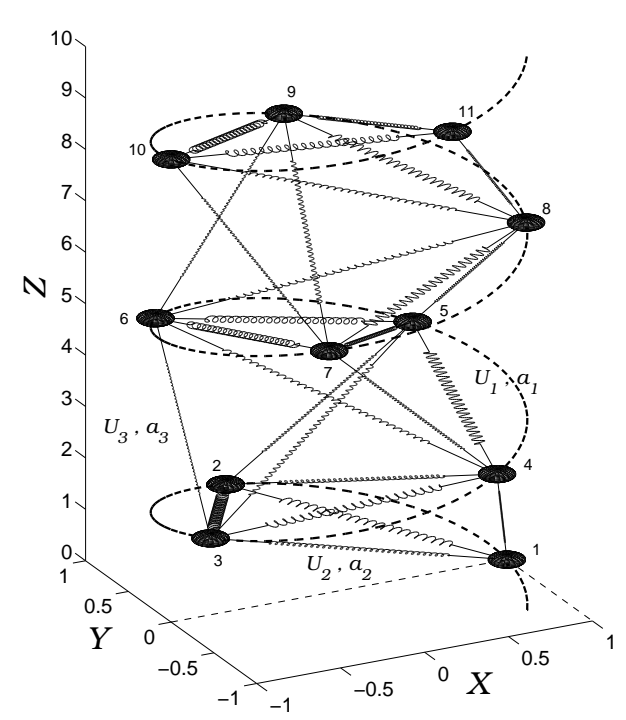

Figure 1. A fragment of the helix backbone consisting of 11 molecules. The geometry of the backbone is in accordance with the structure of $\alpha$ helix, i.e., $\nu=3$ and $\phi=100^{\circ}$. The intermolecular interactions $U_{j}$, $j=1,2,3$, are schematically shown by springs of different thickness. The thicker is a spring the stronger is stiffness of the bond. Undistorted length of the bonds is shown by the dimensionless distances $a_{j}=$ $D_{j} / R_{0}$ 's.

Alternatively, the helix can be described by other three parameters, namely by the distances between (i) the nearest molecules $\left(D_{1}\right)$, (ii) the second neighbours $\left(D_{2}\right)$, and (iii) the $n$th and $(n+\nu)$ th molecules $\left(D_{\nu}\right)$. The distance $D_{j}$ that connects the $n$th and $(n+j)$ th vertices of the backbone is $D_{j}=$ $\sqrt{2[1-\cos (j \phi)]+j^{2} h^{2}} R_{0}, j=1,2, \nu$. There is one-to-one correspondence between the two sets of parameters: $\left\{R_{0}, \phi, h\right\}$ and $\left\{D_{1}, D_{2}, D_{\nu}\right\}[13]$.

The third set of parameters which can also be adopted for the description of helical structure is $\left\{D_{1}, \psi, D_{\nu}\right\}$ with $\psi$ being the valence angle between the nearest-neighbour bonds. Again, the relation between the parameter sets $\left\{R_{0}, \phi, h\right\}$ and $\left\{D_{1}, \psi, D_{\nu}\right\}$ can be given explicitly [13].

Since the regular helix structure is given uniquely by three geometric parameters, we need to consider three types of intermolecular interactions that stabilize this structure. One of these has to be introduced, as usual, between the nearest neighbours along the helix chain and it may be referred to as valence bonds. The second one couples the molecules situated along the longitudinal direction of the chain and it forms secondary structure of the helix. Thus, in protein these interactions are soft and called hydrogen bonds. However, in order to have a stable helix backbone, besides these two we have to consider some third type of the bonds. For instance, it can be the three-particle interaction fixing a certain valence angle $\psi$. The simplest and straightforward way to take into account this fact is to consider the interaction between the $n$th and $(n+2)$ th molecules, i.e., the secondneighbour coupling. In this respect, such a helix backbone will be the most simple generalization of the 1D FPU chain to three dimensions.

\section{Basic equations}

Let $M$ be the mass of molecules and $K$ the characteristic stiffness of the intermolecular forces, $x_{n}, y_{n}$, and $z_{n}$ the coordinates of an instant displacement of 
the $n$th molecule from its equilibrium position. Then the coordinates of the vector $\mathbf{q}_{n}$ describing the displacement of the $n$th molecule are $q_{1 n}=\cos (n \phi)+x_{n} / R_{0}$, $q_{2 n}=\sin (n \phi)+y_{n} / R_{0}$, and $q_{3 n}=n h+z_{n} / R_{0}$. The total Hamiltonian of the helix backbone with the three types of interactions, that link the first and second neighbours as well as the nearest neighbours along the longitudinal direction of the backbone ( $\nu$-neighbours), can be written in a standard manner through the intermolecular potentials $U_{j}\left(r_{j n}\right), j=1,2, \nu$, depending only on the distances $r_{j n}=\left|\mathbf{q}_{n+j}-\mathbf{q}_{n}\right|$ between the $n$th and $(n+j)$ th molecules. The functions $U_{j}(r), j=1,2, \nu$, are assumed of the standard form (like the Lennard-Jones or Morse potential) with $U_{j}\left(a_{j}\right)=0$ and $U_{j}^{\prime}\left(a_{j}\right)=0$ where the prime denotes differentiation. However, in order to find soliton solutions, it is convenient to introduce the set of new variables $\eta_{n}, \theta_{n}$, and $\beta_{n}$ according to $q_{1 n}=\left(1+\eta_{n}\right) \cos \left(n \phi+\theta_{n}\right), q_{2 n}=\left(1+\eta_{n}\right) \sin \left(n \phi+\theta_{n}\right)$, and $q_{3 n}=n h+\beta_{n}$ The generalized coordinate $\eta_{n}$ describes the radial displacement of the $n$th molecule from the cylinder surface which spans the helix backbone when its molecules are situated at the equilibrium positions. The second coordinate $\theta_{n}$ describes the azimuthal deviation of the $n$th molecule from its equilibrium position, and $\beta_{n}$ is the $Z$ coordinate of the displacement. Then the distance $r_{j n}$ is given by $r_{j n}^{2}=\left(1+\eta_{n}\right)^{2}+\left(1+\eta_{n+j}\right)^{2}-2\left(1+\eta_{n}\right)\left(1+\eta_{n+j}\right) \cos \left(j \phi+\theta_{n+j}-\theta_{n}\right)+\left(j h+\beta_{n+j}-\beta_{n}\right)^{2}$ and the corresponding equations of motion become

$$
\begin{aligned}
\frac{\mathrm{d}^{2} \eta_{n}}{\mathrm{~d} \tau^{2}}= & \left(1+\eta_{n}\right)\left(\frac{\mathrm{d} \theta_{n}}{\mathrm{~d} \tau}\right)^{2}-\sum_{j} W_{j}\left(r_{j, n-j}\right)\left[1+\eta_{n}-\left(1+\eta_{n-j}\right) \cos \left(j \phi+\theta_{n}-\theta_{n-j}\right)\right] \\
& +\sum_{j} W_{j}\left(r_{j n}\right)\left[1+\eta_{n}-\left(1+\eta_{n+j}\right) \cos \left(j \phi+\theta_{n+j}-\theta_{n}\right)\right] \\
\frac{\mathrm{d}^{2} \theta_{n}}{\mathrm{~d} \tau^{2}}= & \frac{1}{1+\eta_{n}}\left[-2 \frac{\mathrm{d} \eta_{n}}{\mathrm{~d} \tau} \frac{\mathrm{d} \theta_{n}}{\mathrm{~d} \tau}+\sum_{j} W_{j}\left(r_{j n}\right)\left(1+\eta_{n+j}\right) \sin \left(j \phi+\theta_{n+j}-\theta_{n}\right)\right. \\
& \left.-\sum_{j} W_{j}\left(r_{j, n-j}\right)\left(1+\eta_{n-j}\right) \sin \left(j \phi+\theta_{n}-\theta_{n-j}\right)\right], \\
\frac{\mathrm{d}^{2} \beta_{n}}{\mathrm{~d} \tau^{2}}= & \sum_{j}\left[W_{j}\left(r_{j n}\right)\left(j h+\beta_{n+j}-\beta_{n}\right)-W_{j}\left(r_{j, n-j}\right)\left(j h+\beta_{n}-\beta_{n-j}\right)\right]
\end{aligned}
$$

with $W_{j}\left(r_{j n}\right)=U^{\prime}\left(r_{j n}\right) / r_{j n}$. Here the time is scaled by $\tau=\omega_{0} t, \omega_{0}=\sqrt{K / M}$, and the summation is performed over $j=1,2, \nu$. It is important that these equations are easily reduced to the standard $1 \mathrm{D}$ chain with the nearest-neighbour interactions if we put $\phi=2 \pi$ and $j=1$ or to the zigzag backbone if $\phi=\pi$ and $j=1,2$ [14].

\section{Soliton profiles}

The analysis of the equations of motion (1)-(3) in the small-amplitude limit shows that the frequency spectrum of the helix chain consists of one optical zone that corresponds to radial $\left(\eta_{n}\right)$ oscillations of the backbone and two acoustic zones describing the torsional $\left(\theta_{n}\right)$ and longitudinal $\left(\beta_{n}\right)$ oscillations. We assume that soliton solutions have a moving permanent profile that propagates with dimensionless 
velocity $s=v / v_{0}$ where $v_{0}=(K / M)^{1 / 2} R_{0}$ is the characteristic velocity of smallamplitude waves propagating along the backbone. Next, we put $\eta_{n}=\eta(n h-s \tau)$, $\theta_{n}=\theta(n h-s \tau)$, and $\beta_{n}=\beta(n h-s \tau)$. Assuming that the wave profile smoothly varies from site to site, we approximate the first and second time derivatives of $\eta_{n}$ by the simplest spatial difference derivatives as follows:

$$
\begin{aligned}
\mathrm{d} \eta_{n} / \mathrm{d} \tau & =-s \eta^{\prime}(n h-s \tau) \simeq-s\left(\eta_{n+1}-\eta_{n-1}\right) / 2 h \\
\mathrm{~d}^{2} \eta_{n} / \mathrm{d} \tau^{2} & =s^{2} \eta^{\prime \prime}(n h-s \tau) \simeq s^{2}\left(\eta_{n+1}-2 \eta_{n}+\eta_{n-1}\right) / h^{2}
\end{aligned}
$$

Since the torsional and longitudinal small-amplitude waves are of the acoustic type, we need to take into account the dispersion that arises from the discreteness of the backbone. More precisely, we represent the time derivatives of $\theta_{n}$ and $\beta_{n}$ by differences that additionally contain higher-order spatial difference derivatives chosen in such a way that they cancel the higher expansion terms while passed to the continuum limit. Thus, in terms of the relative displacements $\varphi_{n}=\theta_{n+1}-\theta_{n}$ and $\rho_{n}=\beta_{n+1}-\beta_{n}$ one can write

$$
\begin{aligned}
\mathrm{d} \theta_{n} / \mathrm{d} \tau & \simeq s\left(\varphi_{n+1}-5 \varphi_{n}-2 \varphi_{n-1}\right) / 6 h \\
\mathrm{~d}^{2} \theta_{n} / \mathrm{d} \tau^{2} & \simeq-s^{2}\left(\varphi_{n+1}-15 \varphi_{n}+15 \varphi_{n-1}-\varphi_{n-2}\right) / 12 h^{2}, \\
\mathrm{~d}^{2} \beta_{n} / \mathrm{d} \tau^{2} & \simeq-s^{2}\left(\rho_{n+1}-15 \rho_{n}+15 \rho_{n-1}+\rho_{n-2}\right) / 12 h^{2} .
\end{aligned}
$$
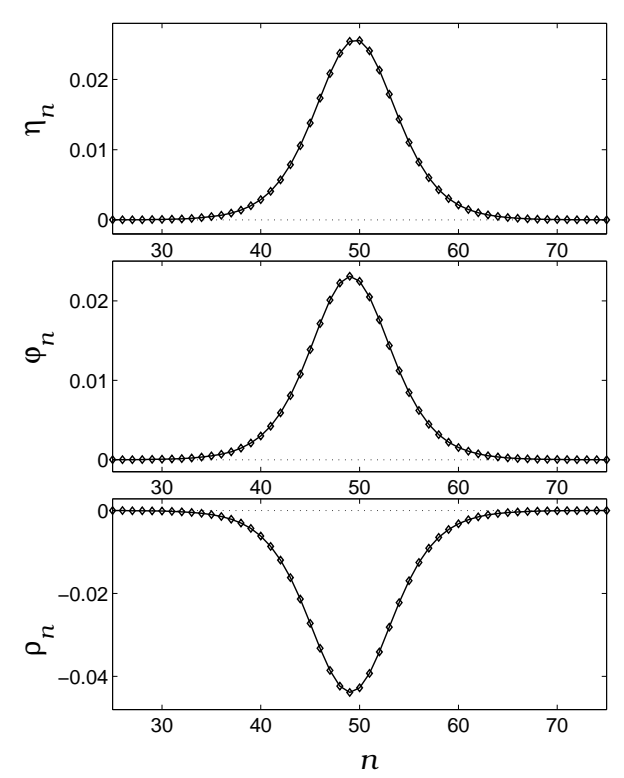

Figure 2. Three-component profile of the soliton in $\alpha$-helix with the anharmonicities $\gamma_{1}=\gamma_{2}=0$ and $\gamma_{3}=1$ found numerically by solving the discrete equations (dashed line) and at the final instant $\tau=28062.45$ when the soliton has passed 100000 chain sites (solid line). The initial velocity of the soliton was $s=1.05 s_{l}$.
Now we replace the time derivatives in equations (1)-(3) by the spatial differences (4)-(8) and obtain discrete equations for the displacements $\eta_{n}, \varphi_{n}$, and $\rho_{n}$ that can be solved numerically, for instance, by minimization techniques [13]. For an appropriate choice of velocity $s$ and sufficiently strong anharmonicity $\gamma_{\nu}$ defined as a coefficient at the cubic term of expansion of the longitudinal soft interaction, the solution of these discrete equations gives three-component bell-shaped profiles shown in figure 2 by dots. The next step in studying soliton solutions to our problem is to use the bell-shaped profiles found by minimization as initial conditions for numerical simulations of the original equations (1)-(3). Then the final profiles of the variables $\eta_{n}(\tau), \theta_{n}$ (or $\varphi_{n}$ ), and $\beta_{n}$ (or $\rho_{n}$ ) obtained under the simulations at sufficiently long 
times $\tau$ allows us to conclude whether or not the initial profiles is a stable soliton solution. In this way we have studied the three-component soliton solution shown in figure 2 by dots in the case of the $\alpha$-helix backbone. For the existence of the soliton solutions only a hard anharmonicity of soft hydrogen bonds is sufficient, it must counterbalance the geometrical anharmonicity. Therefore we took into account only the anharmonicity of the third neighbours $(\nu=3)$. The numerical solution has shown that at weak anharmonicity, when $\gamma_{3}=0.1$, there are no soliton solutions while for the value $\gamma_{3}=1$, the soliton solutions exist only with supersonic velocities in the narrow segment $1<s / s_{l}<1.065$ where $s_{l}=v_{l} / v_{0}$ is the dimensionless velocity of longitudinal sound. As illustrated by figure 2, when the initial speed was $s=1.05 s_{l}$, the simulation of equations (1)-(3) has demonstrated that after passing over 100000 chain sites, the final soliton shape shown by solid lines perfectly coincides with the initial profile shown by dots. Moreover, simulations of the head-in collision of these solitons has confirmed their stability.

\section{A Helical Ratchet}

By analogy with solitons in condensed matter physics, where mechanical devices have proved fruitful in demonstrating their remarkable properties [15] (see, e.g., Scott's model [16] experimentally demonstrating the propagation of topological solitons), mechanical ratchet models could also be useful for analyzing the physics of their function. In the simplest terms, let us restrict ourselves to a system consisting of only two bodies as shown schematically in figure 3 . The experimental setup consists of two massive bars $A$ and $B$ with masses $M_{A}$ and $M_{B}$ which are linked by three springs 1,2 , and 3 . The geometrical configuration shown in figure 3 mimics a left-handed helical structure (e.g., springs 1 and 2 could model the helically coiled $\alpha$-helices of the $\gamma$-subunit of $\mathrm{F}_{1}$-ATPase [17]). Spring 3 with a stiffness constant $K$ supports bar $A$ at a certain equilibrium distance from bar $B$. The action of gravity on bar $A$ is balanced with the compression force of spring 3 , so that the equilibrium distance between the bars occurs at $h-\Delta h$ where $\Delta h=M_{A} g / K R_{0}$ with $g$ being the gravitational acceleration. In this equilibrium state, springs 1 and 2 are undistorted and we denote their equilibrium length by $r_{0}$. Let $\phi$ be the angle between the orientations of bars $A$ and $B$ when the system is found in the equilibrium state. Then the angle $\phi$ depends on other geometrical parameters through the relation $r_{0}=\sqrt{4 \sin ^{2}(\phi / 2)+\left(h-M_{A} g / K R_{0}\right)^{2}}$.

The supporting plate in the setup creates a sliding friction for rotatory motion of bar $B$. This friction depends on the direction of the normal component of the force applied from the side of bar $A$ through all springs 1,2 and 3. Any forcing that exceeds the friction of rest between bar $B$ and the supporting plate causes the rotation of this bar on the plate. The ratchet will work, as we intuitively expect, to one direction only, namely to the clockwise direction (when viewed from above) because the rotation of bar $A$ in this direction causes the tension of springs 1 and 2 whereas the rotation in the opposite direction compresses these springs. As a result, the normal component of the spring force applied to bar $B$ in the vertical direction 


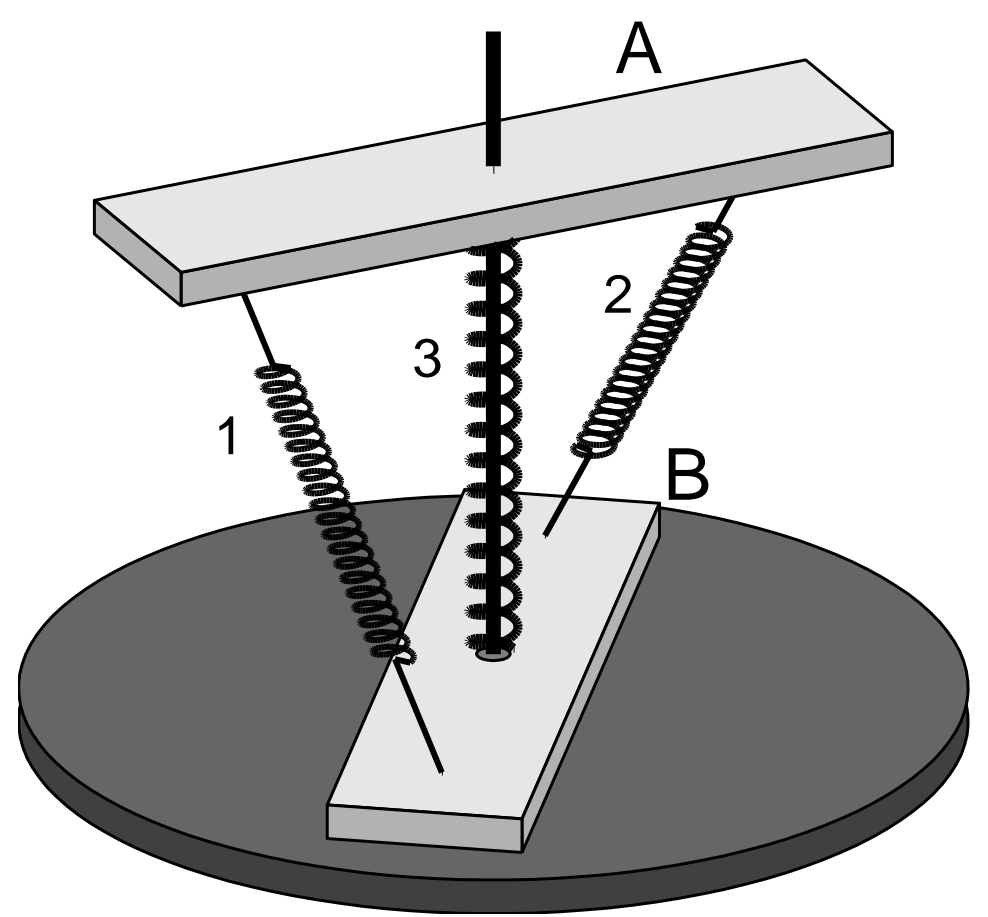

Figure 3. The experimental setup: two bars $A$ and $B$ are linked by two compact springs (1 and 2) forming together a left-handed helix. Central spring (3) supports the upper bar. The supporting bottom plate creates a sliding friction for the lower bar. A driving oscillating or fluctuating force is applied to bar $A$ by, for example, hitting it with a hammer from various directions. Coloured noise was created by the sound from a fog horn acting on a horizontal membrane glued on top of bar $A$. In all cases of forcing, the helix was observed to rotate only in one direction.

will be directed upwards in the former case and downwards in the latter case. This change of the applied force will lead to the corresponding behaviour of the magnitude of reaction and therefore of friction.

Let us denote the angular deviations of bars $A$ and $B$ from the equilibrium positions by $\theta_{A}(t)$ and $\theta_{B}(t)$. Except these two variables, we also consider the vertical displacement $z(t)$ of bar $A$ from the height $h$. Using the arguments given above, Newton's second law yields the following system of three dynamical equations with respect to the variables $\theta_{A}, \theta_{B}$ and $z$ :

$$
\begin{aligned}
M_{A} \ddot{\theta}_{A} & =\kappa\left(1-r / r_{0}\right) \sin \left(\phi+\theta_{B}-\theta_{A}\right)+F_{t} / R_{0}, \\
M_{B} \ddot{\theta}_{B} & =-\kappa\left(1-r / r_{0}\right) \sin \left(\phi+\theta_{B}-\theta_{A}\right)-\Gamma N \dot{\theta}_{B} / g, \\
R_{0} M_{A} \ddot{z} & =N-\left(M_{A}+M_{B}\right) g+F_{n} .
\end{aligned}
$$

Here $F_{n}$ and $F_{t}$ are the normal and tangential components of an external force applied to bar $A$ whereas bar $B$ is subject only to the sliding friction with $\Gamma$ being a damping coefficient. The friction force acting on bar $B$ is assumed proportional to its angular velocity $\dot{\theta}_{B}$ and the reaction $N$ acting from the side of the supporting plate is $N=M_{B} g-R_{0}\left[\kappa\left(1-r_{0} / r\right)(h+z)+K z\right]$. The instant length $r$ of springs 1 
and 2 is given by $r=\sqrt{2-2 \cos \left(\phi+\theta_{B}-\theta_{A}\right)+(h+z)^{2}}$. For simplicity, the strain energy of all the springs in the system of equations (9) is given in terms of harmonic potentials.

A symmetric forcing is applied to the upper bar and the lower bar may be considered as a loaded part of the device that carries out usable work. The heart of this machine is the asymmetric dependence of the friction on forcing due to the helical structure. Intuitively, under the action of the driving force $F_{n}(t)$ one can expect that when $F_{n}>0$ (the applied force is directed upwards), bar $B$ rotates clockwise $\left(\dot{\theta}_{B}<0\right)$, and vice versa, when $F_{n}<0$ (the force is directed downwards), the bar rotates anti-clockwise $\left(\dot{\theta}_{B}>0\right)$. On average, the angular velocity $\dot{\theta}_{B}$ will accumulate the angular drift of bar $B$ in the clockwise direction and molecular dynamics simulations have confirmed such a clockwise drift motion.

\section{Conclusions}

It is an attractive point of view to study the transport of vibrational energy in biopolymers such as protein on the basis of $1 \mathrm{D}$ nonlinear lattice models. The first attempt in this direction was formulated by Davydov and Kislukha [4] (see also the book [5] and the review paper [6] and references therein) who suggested that the intramolecular vibrational amide-I mode could be self-trapped through its interaction with deformation in the protein structure. Further, Davydov et al [18] and Scott [19] generalized this theory by taking the dipole-dipole coupling between the three spines into account. On the other hand, Yomosa [7] modelled the energy transfer in protein, essentially applying the 1D FPU model. The present paper also focuses only on the large-amplitude supersonic acoustic solitons, but in 3D helices the backbone of which is stabilized by three types of intermolecular bonds. Alternatively, instead of the second-neighbour intermolecular bonds, a three-body force that fixes the valence angle $\psi$, could be considered. Any of these force constraints is sufficient for a helix backbone to have a single ground state. The 3D helical model studied in the present paper may be considered to be the simplest generalization of the 1D FPU chain to three dimensions including all degrees of freedom of the chain molecules.

Three-component non-topological solitons have been shown to exist and propagate with supersonic velocities in the helix backbone with any number of spines. Similarly to the 1D case, for the existence of the soliton solutions, the presence of anharmonicity, at least, in the longitudinal (hydrogen) bonds connecting each $n$th and $(n+\nu)$ th molecules, is a necessary condition. However, compared to the 1D FPU model, the existence of stable soliton solutions in the 3D helix backbone appears to be more limited. First, they exist if the anharmonicity of the $\nu$-neighbour forces (longitudinal bonds) is sufficiently strong. Second, the segment of admissible (supersonic) velocities of solitons is always finite. It is quite narrow and for all velocities from this segment, the soliton propagation is uniform with retaining velocity and profile. However, when the solitons collide, their behaviour after collision depends on the velocity. At the lower edge of the soliton velocity spectrum, the soliton collision is elastic whereas for higher velocities, at the upper edge, the solitons collide inelasti- 
cally, with radiation of small-amplitude waves. In summary, we conclude that in a 3D isolated helix backbone the three-component solitons, describing supersonic pulses of longitudinal backbone compression (accompanied by a localized radial bulge and a torsional stretching), still exist as dynamically stable objects. However, the range of their existence is more limited if compared to the 1D FPU lattice.

Another interesting problem considered in this paper is whether the helical asymmetry can induce rectifying zero-mean oscillating or fluctuating rotary motions, similar to thermal ratchets. To this end, we have constructed a simple two-body helical model that supports ratchet rotatory motion. This ratchet mechanism might be of interest for studying the $\mathrm{F}_{1}$-ATPase motor [17].

Acknowledgements. This work was partially carried out with the financial support from the European Economic Community (EEC) under the INTAS Grant No. 96-158. Two of us (A. V. Z. and A. V. S.) would also like to express his gratitude to the MIDIT Center and the Department of Mathematical Modelling of the Technical University of Denmark.

\section{References}

1. Fermi E., Pasta J., Ulam S. Collected Works of Enrico Fermi. Vol. 2. Chicago, 1965, p. 978.

2. Zabusky N.J., Kruskal M.D. Interaction of solitons in a collisionless plasma and the recurrence of initial states. // Phys. Rev. Lett., 1965, vol. 15, p. 240-243.

3. Toda M. Theory of Nonlinear Lattices. Berlin, Springer, 1978.

4. Davydov A.S., Kislukha N.I. Solitary excitations in one-dimensional molecular chains. // Phys. Stat. Sol. B, 1973, vol. 59, p. 465-470.

5. Davydov A.S. Solitons in Molecular Systems. Dordrecht, Reidel, 1985.

6. Scott A.C. Davydov's soliton. // Phys. Rep., 1992, vol. 217, p. 1-67.

7. Yomosa S. Solitary excitations in muscle proteins. // Phys. Rev. A, 1985, vol. 32, p. $1752-1758$.

8. Olsen O.H., Lomdahl P.S., Kerr W.C. Localized excitations in a three-dimensional nonlinear model of a 310 -helix. // Phys. Lett. A, 1989, vol. 136, p. 402-408.

9. Cadet S. Transverse envelope solitons in an atomic chain. // Phys. Lett., 1987, vol. 121, p. $77-82$.

10. Feynman R.P., Leighton R.B., Sands M. The Feynman Lectures on Physics. vol. 1. Massachusetts, Addison-Wesley, Reading, 1966, p. 46.

11. Magnasco M.O. Forced thermal ratchets. // Phys. Rev. Lett., 1993, vol. 71, p. 1477.

12. Astumian R.D., Bier M. Fluctuation driven ratchets: molecular motors. // Phys. Rev. Lett., 1994, vol. 72, p. 1766-1769.

13. Christiansen P.L., Zolotaryuk A.V., Savin A.V. Solitons in an isolated helix chain. // Phys. Rev. E, 1997, vol. 56, p. 877-889.

14. Zolotaryuk A.V., Christiansen P.L., Savin A.V. Two-dimensional dynamics of a free chain with a secondary structure. // Phys. Rev. E, 1996, vol. 54, p. 3881-3894.

15. Remoissenet M. Waves Called Solitons: Concepts and Experiments. Berlin, Springer, 1996.

16. Scott A.C. A nonlinear Klein-Gordon equation. // Am. J. Phys., 1969, vol. 37, p. 5261. 
17. Noji H., Yasuda R., Yoshida M., Kinosita Jr. K. Direct observation of the rotation of F1-ATPase. // Nature, 1997, vol. 386, p. 299-302.

18. Davydov A.S., Eremko A.A., Sergienko A.I. Solitons in $\alpha$-helical protein molecules. // Ukr. Fiz. Zh. (Ukrainian Phys. J.), 1978, vol. 23, p. 983-993 (in Russian).

19. Scott A.C. Dynamics of Davydov solitons. // Phys. Rev. A, 1982, vol. 26, p. 578-595.

\title{
Солітони і храповикові рухи у спіралях
}

\author{
А.В.Золотарюк, П.Л.Крістіонсен, Б.Норден, А.В.Савін
}

1 Інститут теоретичної фізики ім. М.М.Боголюбова, 252143 Київ

2 Факультет математичного моделювання, Технічний університет Данії, DK-2800 Лінгбі, Данія

3 Факультет фізичної хімії, Університет технології Чалмерса, S-412 96 Ґотенбург, Швеція

4 Державний інститут проблем фізики і технології, 119034 Москва, Російська Федерація

Отримано 4 вересня 1998 р.

Показано, що найбільш просте і безпосереднє тривимірне узагальнення стандартного одновимірного молекулярного ланцюжка із взаємодією найближчих сусідів має вигляд спірального скелета з трьома типами міжмолекулярних взаємодій - ланцюг із вторинною структурою (наприклад, $\alpha$-спіраль у біології). Виведено систему зв'язаних нелінійних польових рівнянь стосовно поперечних і поздовжніх зміщень молекул ланцюжка і знайдені стійкі нетопологічні трикомпонентні солітонні розв'язки. Ці розв'язки описують надзвукові биття поздовжнього стиску, що судпроводжуються локалізованою поперечною опуклістю і локалізованим скрученим розтягом спірального скелета. На прикладі простої механічної моделі показано, що спричинена спіральністю скелета асиметрія виправляє середні осцилюючі або флюктуюючі рухи.

Ключові слова: ангармонічні ланцюжки, спіралі, солітони, храповики

PACS: $63.20 . R y, 63.20 . P W, 87.15 .-v$ 